\title{
DAS BESETZEN VON BEGRIFFEN: KOMMUNIKATIVE STRATEGIEN UND GEGENSTRATEGIEN IN DER UMWELTDISKUSSION
}

\section{Ulrike Haß}

\section{Was heißt "Besetzen von Begriffen" in der Umweltdiskussion?}

In der nunmehr zwanzig Jahre andauernden öffentlichen Diskussion über Umweltprobleme herrschen kommunikative Strategien vor, die sich mit dem, was Hans Maier und Kurt Biedenkopf seinerzeit "Besetzen von Begriffen"1 nannten, nur noch partiell identifizieren lassen. Das liegt einmal an der fachsprachlichen Herkunft der meisten Schlüsselwörter, für die keine, auf die gesamte Sprachgemeinschaft ausgedehnten, traditionell vertrauten Bedeutungskonzepte existieren, die in politischer Absicht umgepolt werden könnten. Vertraute Konzepte sind bei einigen Ausdrücken höchstens bruchstückhaft in Form assoziativer Aspekte vorhanden, wie bei Kern: 'kernig', 'gesund', bei entsorgen: 'von Sorgen befreien' usw. Solche Assoziationen werden allerdings - im Falle von Kern nachweislich - strategisch eingesetzt.

Zum andern liegt der Fall in der Umweltdiskussion auch deshalb anders als in der von Maier und Biedenkopf ins Visier genommenen politischen Situation, weil diejenigen Sprechergruppen, die sich tatsächlich eher auf traditionelle Begriffskonzepte beziehen wie Gleichgewicht, Kreislauf, ganzheitlich, Natur/natürlich usw., d.h. grob gesagt Sprecher der Ökobewegung, nicht in ausreichendem Maße Zugang zu den wichtigen Medien haben. Bevor mir jetzt Widerspruch entgegenschallt, gebe ich zu bedenken, daß die Initiativ- und Schlüsselrolle beim Durchsetzen von Ausdrücken mit bestimmten Inhalten und Perspektivierungen bei der Allianz von staatlicher Verwaltung, Technik und Industrie liegt; diese nämlich produzieren in Übereinstimmung ihrer Interessen jene Programme, Gesetze, Verordnungen, Risikostudien und Gutachten, auf die die Publikumsmedien letztlich nur reagieren. ${ }^{2}$

Von echten Begriffsbesetzungen kann man nur insofern sprechen, als "grüne" Fahnenwörter, seien sie gemeinsprachlich vertraut wie die oben genannten Kreislauf usw. oder fachsprachlich unvertraut wie Recycling, immer wieder für Werbezwecke eingesetzt und damit die Zuordnung dieser Ausdrücke zu grundsätzlich anderen Sprecherintentionen konventionalisiert wird: Kreislauf wurde

\footnotetext{
${ }^{1}$ Siehe beider Beiträge in: Heringer 1982.

${ }^{2}$ Dies ist durch textsortenbezogene Wortschatzvergleiche empirisch belegbar.
} 
als Brennstoffkreislauf zum Hauptargument für die WAA Wackersdorf (die eben nötig sei, um den Brennstoffkreislauf zu schließen) ${ }^{3}$. Beispiele für die Verwendung von Natur-, bio-, öko-, umweltfreundlich usw. in der Produktwerbung kennt jeder. Im Prinzip das gleiche geschieht bei der Verwendung der Ausdrücke Gleichgewicht oder Ausgleich, wenn das diesen Bezeichnungen implizite naturphilosophische Konzept - Stichwort Carl von Linné: Oeconomia naturae, 1749 -, das das Gleichgewicht des Naturhaushalts mit der von Gott geschaffenen Harmonie begründet, wenn also dieses Konzept neuerdings auf das Verhältnis von Ökologie und Ökonomie angewendet wird, so als sei das betriebswirtschaftliche Prinzip der Gewinnorientierung Bestandteil der ohnehin unaufhebbaren Harmonie der Natur. ${ }^{4}$

Diesen Fällen von Begriffsbesetzungen liegt allerdings kein einheitliches, durchgehendes ideologisches Programm zugrunde; umweltfreundlich oder recyclinggerecht bedeutet bei fast jeder Realisierung etwas anderes; sie sind gar nicht dauerhaft besetzt, sie werden ihrer Wertungsfunktion wegen nach Bedarf benutzt.

Im folgenden will ich mich auf diejenigen kommunikativen Strategien konzentrieren, die an den Hauptkonfliktpunkten der Umweltdiskussion die entscheidende Rolle spielen, und die von Sprechern selbst thematisiert werden. Es gibt hier nämlich mehr als in früheren politischen Debatten eine Auseinandersetzung um die in ihr eingesetzten kommunikativen Mittel selbst, und es gibt mehr oder weniger erfolgreiche Gegenstrategien.

\subsection{Bedeutungsfestlegung durch Experten}

Im Zusammenhang mit dem aus verschiedenen Wissenschafts- und auch Technikbereichen stammenden Hintergrundwissen wurde zunächst rein fachintern eine Menge fachsprachlicher Ausdrücke eingeführt und für die fachinterne Verwendung definiert.

Seit Beginn der öffentlichen Umweltdiskussion wurden dann mehr und mehr fachintern produzierte Texte durch Experten selbst, durch professionelle Vermittler und vor allem durch Journalisten popularisiert. Während dieses Transfers in den fachexternen Kommunikationsbereich blieben und bleiben Fachausdrücke für naturwissenschaftliche, produktionstechnische und juristische Sach-

\footnotetext{
${ }^{3}$ Die Bedeutungsangaben, die H. Gründler in seinem Aufsatz "Kernenergiewerbung" (in: Heringer 1982:208) zu Brennstoffkreislauf macht, sind sachlich unrichtig. Vgl. den Artikel "Brennstoffkreislauf/Plutoniumwirtschaft/Wiederaufarbeitung". In: Strauß u.a. 1989:456-461.

${ }^{4}$ Erläuterung und Belege von Formeln wie Ausgleich von Ökonomie und Ökologie siehe Strauß u.a.1989:485 ff.
} 
verhalte weitgehend unverändert. D.h. ihre Ausdrucksseite bleibt unverändert; jedoch verändert sich durch den anderen Kommunikationsrahmen, z.B. den politischen Konflikt pro und contra Kernenergie, die Bedeutung vieler (Fach-) Bezeichnungen dahingehend, daß sie vieldeutig oder vage werden und die fachsprachlich bedingte Wertungsneutralität verlieren, die ihnen in der fachinternen Perspektive zugesprochen wird. Juristische Bezeichnungen verlieren die Eigenheit, auf bestimmte, in Gesetzen festgelegte Fälle oder Bereiche beschränkt zu sein. Manche Ausdrücke gewinnen u.U. Schlagwortcharakter. Typische Beispiele für diese Gruppe sind: Altlasten, Brennelement, Entsorgung, Gau, Restrisiko, Spaltprodukt, Störfall, Umweltverträglichkeit.

So wurde Störfall in Abgrenzung zu Unfall erstmals in der Strahlenschutzverordnung von 1976 (in Kraft ab 1.4.1977, inzwischen durch das sog. Strahlenschutzvorsorgesetz ersetzt) ausdrücklich definiert. Diese Bezeichnungsfestlegung wird in den Studienbriefen des "Funkkollegs Mensch und Umwelt" von 1983 wie folgt weitergegeben:

Kernenergietechniker unterscheiden ... zwischen einem Unfall und einem Störfall, wobei das Hauptunterscheidungsmerkmal die Belastung durch radioaktive Strahlung ist, die in die Umgebung des Kernkraftwerkes gelangt. Unterhalb eines bestimmten Grenzwertes, der in der Strahlenschutzverordnung festgelegt ist, handelt es sich um einen Störfall, erst oberhalb dieses Grenzwertes spricht man von einem Unfall. (Dahlhoff 1983:264)

Die Klasse der Störfälle wird dann noch einmal unterteilt in eine schwere (Kategorie 'Eil(t)'), eine mittelschwere (Kategorie 'Normal') und eine leichte Kategorie, die nur noch Vorkommnis genannt wird, mit dem Zweck, die Kommunikation zwischen Kernkraftwerk bzw. Chemiebetrieb und Behörden zu regeln. Die Einstufung in die Kategorie ' $X$ ' entscheidet darüber, ob das, was da passiert ist, meldepflichtig ist oder nicht. Meldepflichtig bedeutet meistens auch die Ausweitung der Kommunikation über das Ereignis auf den öffentlichen Bereich, in dem Sprecher sich nicht an die fachintern getroffenen Bezeichnungsfestlegungen halten. Das Besetzen und Besetzthalten der Begriffe Störfall und Unfall funktioniert vor allem in der Kommunikation zwischen Technikexperten, Industrie und Behörden, darüber hinaus nur dort, wo das beanspruchte Definitionsmonopol dieser Sprechergruppen fraglos akzeptiert wird wie in vielen populärwissenschaftlichen Nachschlagewerken ${ }^{5}$ und in Erzeugnissen des sog. Verlautbarungsjournalismus. Da heißt es dann: "Der jährliche Bericht des Bundesinnenministers über besondere Vorkommnisse in Kernkraftwerken unterstreicht einmal mehr: Deutsche Reaktoren arbeiten sicher und sauber. Auch

\footnotetext{
${ }^{5}$ Stellvertretend für viele sei genannt: Ahlheim 1981. Wie funktioniert das? Die Umwelt des Menschen.
} 
1984 gab es weder einen Störfall noch einen Unfall." (MANNHEIMER MORGEN 14.8.1985). Hier wird die fachintern definierte Abgrenzung zwischen Störfall und Unfall stillschweigend vorausgesetzt.

Ein anderes charakteristisches Merkmal der Verwendung von Fachausdrücken in der Umweltdiskussion ist die mit ihnen vorgenommene Perspektivierung, die von den Problemaspekten weg- und auf Aspekte der Problemlösung hinführt. Diesbezügliche Paradebeispiele sind Entsorgung und Entsorgungspark als Ausdrücke, die in extremer Weise beunruhigende Aspekte des Bezugsobjekts ausblenden; weitere Beispiele sind: Altlastensanierung, Reaktorsicherheit, Brennstoffkreislauf, Sonderabfall. Der jahrzehntelange Gebrauch der Bezeichnungen Pflanzenschutzmittel, Schädlingsbekämpfungsmittel bzw. Herbizide, Insektizide, Fungizide usw., mit denen von den Bezugsobjekten prädiziert wird, daß sie gegen etwas Schlechtes gut sind, haben sicher nicht wenig dazu beigetragen, daß die Eigenschaft, schlecht fürs Grund- und Trinkwasser zu sein, bis vor ganz kurzer Zeit kein öffentliches Thema war.

Bei Sonderabfall, einem Verwaltungsfachausdruck aus dem Abfallrecht, besteht die Perspektivierung in der für diese Abfallart vorgesehenen gesonderten oder besonderen Behandlungsweise, ohne daß deren inhaltliche Zusammensetzung in den Blick geriete.

Was ich hier beschreibe, ist unter der Bezeichnung Euphemismus geläufig. Man kann auch sagen, daß hier Sehweisen "besetzt" werden sollen. Euphemismus fasse ich allerdings nicht als einen linguistischen Terminus auf, sondern ich rechne ihn genauso wie die Prädikate verschleiernd und beschönigend zu den eher nicht-linguistischen, sprachthematisierenden Ausdrücken, mit denen kommunikatives Verhalten bewertet wird. Ob ein Ausdruck $X$ abwertend als Euphemismus charakterisiert wird, hängt von Weltbild und Problemsicht des Sprechers ab. Der Euphemismusvorwurf gehört zu den wichtigsten Strategien, die in der öffentlichen Diskussion gegen die Perspektivenvorschriften der Experten eingesetzt werden. Im Falle des Entsorgungsparks in Gorleben war sein Erfolg so groß, daß die Angegriffenen umgehend auf den Ausdruck integriertes Entsorgungszentrum umschalteten; bei der technisch z.T. anderen Konzeption der Anlage in Wackersdorf war dann nur noch von Wiederaufarbeitung mit oder ohne Endlagerung die Rede.

\subsection{Euphemismusvorwurf als Gegenstrategie}

Sprecher, die einen Ausdruck als Euphemismus verworfen haben, können ihn natürlich selbst nicht mehr verwenden. Als Mittel zur aktiven Perspektivenkorrektur werden daher Konkurrenz- und Alternativausdrücke eingeführt, die ein 
Problemthema in einer anderen Perspektive präsentieren und z.T. auch als Fahnenwörter für die eigene Meinungsgruppe fungieren. Beides zusammen liegt bei Giftmüll als Alternativbezeichnung zu Sonderabfall vor. Erfolgreich ist die Propagierung solcher Gegenwörter vor allem über das Medium der nicht rechtsrelevanten Texte in Presse, Rundfunk, Fernsehen, in denen sie wohl aus im weitesten Sinne stilistischen Gründen dem "trockenen" Expertenjargon vorgezogen werden.

\subsection{Metaphorisierungen als Gegenstrategie}

Metaphorisierungen sind in begrenztem Maße dazu geeignet, die Gültigkeit von Bezeichnungs- und Perspektivenfestlegungen zu relativieren und insofern das "Besetzermonopol" der Experten zu brechen. Da gibt es einmal die im Themenbereich bleibenden Metaphorisierungen: Sprecher verwenden einen Ausdruck wie Gau oder Störfall als kritische Charakterisierung für Handlungs- oder Verhaltensweisen mit Bezug auf dasselbe Umweltthema, in dem man mit Gau oder Störfall ein tatsächliches Ereignis bezeichnet (hat).

So sprach man beispielsweise im Zusammenhang mit der Atomkraftwerkskatastrophe in Tschernobyl mehrfach vom Informations-Gau in den Medien.

Oder: Im Zusammenhang mit der Berichterstattung über einen sog. Störfall im Reaktor Hamm lautete der Titel eines Zeitungsartikels "Informations-Störfall. Wie man der Bevölkerung Angst macht", womit Kritik an der Informationspraxis des zuständigen Ministers zum Ausdruck gebracht werden sollte.

Ein die ursprüngliche Bezeichnungsfestlegung noch stärker relativierender Effekt geht von den Metaphorisierungen außerhalb von Umweltthemen aus: Entsorgung der Vergangenheit, die Barschel-Affäre ein Störfall in der demokratischen Gesellschaft, Waldheims Kriegsvergangenheit eine Altlast $^{6}$ - mit solchen Ausdrucksweisen demonstrieren Sprecher, daß sie sich über die von Experten gesetzten Grenzen hinwegsetzen.

\subsection{Etymologische Wortspiele als Gegenstrategie}

Ironisierende Etymologisierungen greifen eine schon in der Reformationszeit virtuos gehandhabte Art strategischer Sprachreflexion auf: die explizite und interessengeleitete Motivierung einer Bezeichnung durch Rückgriff auf (angebliche) Eigenschaften des Bezeichneten. Ein Journalist, der Restrisiko paraphrasiert als "Risiko, das uns den Rest gibt", nimmt eine Neumotivierung vor,

\footnotetext{
${ }^{6}$ Alle in diesem Aufsatz genannten Beispiele sind belegt unter dem jeweiligen Stichwort in: Strauß u.a. 1989.
} 
die auf eine Destruktion der mit dieser Bezeichnung vorgegebenen Perspektive abzielt. Eine irgendwie bleibende Wirkung auf die Semantik eines Ausdrucks können solche vereinzelten Verfahren natürlich nicht erreichen. Allgemein geläufig geworden ist wohl nur die gar nicht immer ironische, sondern ernstgemeinte Remotivierung von entsorgen als ‘sich der Sorgen entledigen’.

\section{Bewertungen ohne Maßstab}

Eine andere Art kommunikativer Strategie bedient sich der in diesem Themenbereich häufigen Einstufungs- und Wertungsausdrücke, deren Besetztsein sich wie schon erwähnt - im Grunde auf das Fixieren einer positiven oder negativen Wertung beschränkt. Der kommunikationsstrategisch wichtige Punkt der Verwendung solcher Ausdrücke ist, daß der Maßstab, den Sprecher und Autoren bei bestimmten Einstufungen und Bewertungen jeweils zugrundelegen, unausgesprochen und verdeckt bleibt oder absichtlich unbestimmt ist. Dadurch haben Hörer und Leser es schwer, die Berechtigung solcher Wertungen zu überprüfen und sich gegebenfalls von ihnen zu distanzieren. Beispiele, für die dies in bestimmten Einzelbedeutungen zutrifft, sind: alternativ, die Alternative, der/die Alternative, Bio-/bio-, Öko-/öko-, der Öko, ökologisch, recyclinggerecht, umweltfreundlich, umweltschädlich u.a.m.

Das Aufkommen dieser Ausdrücke hängt mit der Herausbildung eines neuen Weltbildes Anfang der 70er Jahre zusammen, aus dem sich deutlich andere Maßstäbe für den Umgang des Menschen mit der Natur ergaben. Weil aber diese Maßstäbe innerhalb der öffentlichen Diskussion kaum explizit gemacht werden bzw. nicht in gleichem Maß wie diese Bewertungsausdrücke selbst kursieren, bleiben die Ausdrücke inhaltlich unbestimmt und damit immmer wieder neu "besetzbar". Z.B. wird mit umweltfreundlich und Umweltschutz gerade für solche Produkte geworben, die in einer anderen Perspektive ökologisch besonders bedenklich sind.

Die Gegenstrategie besteht hier in der Explizierung der jeweils zugrundegelegten Bewertungsmaßstäbe und darin, diese Explizierungen auch von anderen Sprechern zu fordern. So hat der Deutsche Bund für Vogelschutz (DBV) Umweltminister Töpfer kritisiert, der Katalysator-Autos als umweltfreundlich bezeichnet hatte, und hatte ihm öffentlich vorgerechnet, wie viele Schadstoffe und $\mathrm{CO}^{2}$ diese im Vergleich zu Bundesbahn und Fahrrad immer noch in die Umwelt abgeben. Die Effektivität dieser Gegenstrategie ist natürlich beschränkt. 


\section{Am längeren Hebel sitzen}

Letzter Punkt der "Besetzungspolitik" in der Umweltdiskussion, auf den ich hinweisen möchte, sind die Textsorten, deren spezielle Möglichkeiten sich einige Sprecherguppen zur Durchsetzung ihrer Rede- und Sichtweisen zunutze machen.

Dazu gehören erstens populärwissenschaftliche Sachbücher, Nachschlagewerke und entsprechende Artikel in neueren Enzyklopädien. Die fachintern vorgenommenen Bezeichnungsfestlegungen werden mittels dieser Texte subtiler und damit wirkungsvoller als mit anderen Medien in einen breiteren, nicht fachgebundenen Kommunikationsbereich transportiert, denn dabei werden das autoritative Prestige und die besonders Nachschlagewerken üblicherweise unterstellte Meinungsneutralität und objektive Informativität gezielt ausgenutzt.

Zweitens sind als besonders durchsetzungsmächtig amtliche Textsorten zu nennen, d.h. solche, deren Wortgebrauch für einen weitreichenden Kommunikationsbereich juristisch verbindlich ist. Der Wortlaut von Gesetzen, Verordnungen usw. ist innerhalb der politischen Kommunikation nicht paraphrasierbar. Z.B. kann in Genehmigungsverfahren, Einspruchsverhandlungen mit Bürgern, Parlamentsanfragen, Rechtsgeschäften usw. von der Terminologie des Atomrechts oder des Abfallrechts nicht abgewichen werden. Die in ihnen vorgenommenen Bezeichnungsfestlegungen sind für einen ausgedehnteren Kommunikationsbereich sanktionsfähig.

Als dritter textueller Faktor ist zu berücksichtigen, daß in öffentlichen Medienorganen Bedingungen herrschen, die den sogenannten Verlautbarungsjournalismus begünstigen. Gerade bei den schwierigen Sachthemen der Umweltdiskussion greifen Journalisten auf die Vorformulierungen der PR-Abteilungen zurück und zitieren aus Hilflosigkeit so getreu wie möglich, was Experten und Politiker auf Pressekonferenzen gesagt haben, und reproduzieren bzw. unterstützen so deren kommunikative Strategien.

Den Sprechergruppen, die einen Monopolanspruch auf die Verwendung bestimmter Ausdrücke und die Festlegung von Bedeutungen erheben, steht also erhebliche mediale Macht zu dessen Durchsetzung zur Verfügung. Die Gegenstrategien anderer Sprechergruppen kommen dagegen kaum an, obwohl sie belegen, daß das Niveau des kommunikativ-sprachlichen Bewußtseins in der öffentlichen Diskussion nie so hoch war wie in der Umweltdiskussion. 


\section{Literatur}

Ahlheim, K.H. 1981 (Hg.). Wie funktioniert das? Die Umwelt des Menschen. 2. Aufl. Mannheim/Wien/Zürich.

Dahlhoff, Th. 1983 (Hg.). Funk-Kolleg Mensch und Umwelt. Bd.II. Frankfurt/M.

Gründler, H. 1982. Kernenergiewerbung. In: Heringer 1982 (Hg.), 203-215.

Heringer, H.-J. 1982 (Hg.). Holzfeuer im hölzernen Ofen. Tübingen.

Strauß, G. / Haß, U. / Harras, G. 1989. Brisante Wörter von Agitation bis Zeitgeist. Ein Lexikon zum öffentlichen Sprachgebrauch (=Schriften des Instituts für deutsche Sprache, 2). Berlin/New York. 